\title{
高低压成套开关设备的优化方法研究
}

夏金华 时悦

湖北省电力装备有限公司

DOI:10.32629/hwr.v3i10.2450

[摘 要] 高低压开关设备在电力系统中负责配电、电气传动以及控制设备的作用。随着我国电网事业的发展, 大量的电力设备、自动控制系统 广泛应用在电力系统中, 高低压开关设备大量增加, 但是高低压成套开关设备在使用过程中, 由于制造工艺等方面存在的缺陷, 导致成套开关的使 用效率比较低, 在使用过程中, 出现误动、拒动现象。因此需要优化高低压成套开关设备设计,提高高低压成套开关的性能和质量,确保供电系统 的安全性和可靠性。

[关键词] 高低压; 成套开关设备; 优化

成套开关设备分为高压成套开关设备和低压成套开关设备。然而在实 际应用过程中, 由于成套开关设备设计、制造方面存在一定的缺陷, 导致成 套开关设备的绝缘性能下降, 影响成套开关设备的运行效率和安全性。

\section{1 国内高低压成套开关设备发展现状}

高低压成套开关设备以及与其相关联的控制设备, 在电力输配电系统 中发挥的作用是极为显著的, 具体在控制、维护、计量、信号传导以及数 据转换等环节体现出来, 其囊括信息量大且与多方面相关联。高低压成套 开关设备技术水平的优劣, 关系着整个电力系統的运转效率与质量, 也关 系着输配电系统操控人员与电力设备自体的安全性。相关资料记载国内每

下面将具体分析输变电工程施工策划方案重点环节施工质量控制方 式。

绝缘件: 最主要的是要避免绝缘件出现起层、起泡等问题, 为此, 优化 实施控制人员需要监督设备生产厂家完成绝缘纸板胶纸布置操作; 还需要 监督设备生产厂家完成热压操作, 并控制其热压温度, 这是因为温度不均 匀可能会导致起层、起泡现象; 需要监督设备生产厂家完成表面清洁、焊 接、毛刺处理工作, 以此来确保操作质量 ${ }^{[4]}$ 。

铁心: 铁心叠装操作中应用到的硅钢片质量是铁心制作的关键点, 同 时需要测量铁心油道绝缘电阻数值, 避免在后期出现电阻通路问题之后再 返工; 检查铁心的波浪程度, 以此来确保铁心捆绑的紧实程度。

线圈: 需要重点关注换位导线在焊接之前的处理。具体需要应用脱漆 剂对换位导线进行脱漆处理, 同时还需要确保脱漆的干净程度, 避免内部 出现残渣导致局部过热; 在对线圈进行压紧处理之后, 需要检查换位导线 的绝缘性能, 确保不存在短路位置; 高场强区制作应用整张厚纸板, 因此需 要检查纸板是否存在起层问题。

设备干燥: 设备干燥需要重视的是总压紧力控制, 具体需要在检查过 程中, 结合设计图纸、施工标准对具体数值进行对比分析, 以此来确保设备 干燥效果符合实际要求。

2. 4 加强信息沟通

优化实施控制小组需要进行良好的信息沟通, 就优化实施控制工作进 展情况进行及时汇报, 在发生质量问题之后需要就问题和解决情况在小组 内公告, 以此来提高信息的共享性和应用有效性, 通过对比分析管理数据 的方式, 来做好优化实施控制工作。

同时, 还需要做好优化实施控制资料记录工作, 优化实施控制资料不 但包括优化实施控制全过程, 还包括问题和解决方式的全过程, 因此, 在开 展优化实施控制工作之前, 电力企业需要组织优化实施控制人员学习统一 的资料记录方式, 同时了解信息收集和归档的要求 ${ }^{[5]}$ 。此外, 优化实施控制
年电装机新增率高于 $8 \%$ 以上, 业内人士预测到 2020 年, 国内电力产业发展 领域装机容量将高于 8 亿kW。

\section{2 高低压成套开关设备的优化分析}

2.1 电路优化方案

在高低压成套开关设备的优化设计中, 对主电路的优化设计需要通过 多方面的因素进行分析, 其主要体现在以下几个方面:

2.1.1确定负荷

在主电路的优化设计中, 需要确定负荷的特性, 以便满足系统的使用 要求, 在负荷的确定因素中, 需要考虑容量、供电距离、分布以及设备等因

人员需要定期将每天、每周、每月、每季度、紧急报告及时送到优化实施 控制小组办公室, 管理人员在核查之后还需要发送给业主, 另业主及时了 解优化实施控制工作进展情况, 并且要确保各方信息沟通的及时性和有效 性, 这也可以方便业主、设备生产厂家、电力企业日后查找和应用资料数 据。通过规范优化程序, 能够提高优化进展的规范程度, 进而确保优化数据 的准确性。开展输变电工程施工方案进行可实施性优化最重要的便是优化 组织, 为此, 需要构建一个效率高、优化严谨、优化合理的阻止结构, 并对 负责优化方案可实施性优化的人员进行合理划分, 对优化工作进行合理划 分, 同时制定权责制度, 进而组织相关的优化活动, 在完成优化之后, 收集 统计优化数据, 对优化数据进行分析, 并结合优化方案的运行条件和需求 对优化结果进行深入分析, 进而得到最终的优化结论。

\section{3 结束语}

开展关于优化输变电工程可实施性的一种策划方案研究可知, 为了做 好优化输变电工程施工策划方案可实施性工作, 需要分别做好前期准备工 作和施工过程控制工作, 同时在此过程中不断探索针对性管理模式, 全面 发挥管理模式的管理作用, 并构建管理系统, 通过先进的安装技术和方式, 以及专业的质量监管控制方式, 为电网工程的效率开展奠定基础。

\section{[参考文献]}

[1]韩文军,魏玲.输变电工程典型设计对电网工程设备制造和材料加 工影响的研究[J].中国新技术新产品,2015,(18):7-9.

[2]王振明,董玉震,宋华松.将物联网技术引入特高压工程管理[J].项 目管理评论,2016,(01):65-67.

[3]输变电工程研究课题汇总 [J].国家电网,2019,(03):133-138.

[4]梁旭明, 丁燕生,陈秀娟. $750 \mathrm{kV}$ 交流输变电工程设备的国产化 [J]. 电 力建设,2016,(08):1-5.

[5]齐胜涛.特高压输电工程的可听噪声及其降低措施[J].河北电力技 术,2016,35(03):63. 
素, 设计合理的电压供给方案, 以此来提升设备的功率因数。其主要优化措 施如下：(1)需要满足设备长期和短期运用的系数以及季节性的运用系数, 最大程度的保障系统的使用性能; (2)需要充分的考虑环境因素, 需要保证 系统的短路电流和接地不会对设备的运行状态产生影响; (3)在设计中要注 重电路的排布问题, 满足建筑的设计需求, 保障电路的适用性; (4)需要优化 参数; (5)需要通过元件以及柜体来满足用户的需求。总之, 只有对主电路 进行优化设计, 才可以最大程度的保障系统的使用性能。

\section{1.2 辅料的优化方案}

在高低压成套开关设备的优化中, 需要对辅料进行优化设计, 在设备 的运行中, 母排、电缆以及壳体均对设备的运行具有较大的影响。如母排 弧形导线具有铜和铝两种常见的形式和规格, 在优化设计过程中, 需要根 据工程和用户的使用需求进行合理的配置, 在设计的过程中, 要保障用户 的过载裕度, 使其在保证使用性能的基础上, 可以最大程度的节约成本。如 在高低压成套开关设备的设计中, 对于电流较大的线路, 可以采用多根铜 排并联的方式进行设计, 这样可以提升设备的整体载流能力, 同时可以节 约开支, 提升系统的整体性能。

\subsection{3符合环境的优化设计}

在高低压成套开关设备的优化设计中, 需要综合的考虑环境因素, 以 便最大程度的保障设备的使用性能。其主要体现在以下几个方面: (1)对于 粉尘或者带电粒子相对较多的环境, 可以采用密封性好以及体积相对较小 的元件, 其可以提升设备的整体运用性能; (2)对于海拔较高或者震动性较 强的环境, 可以采用具有抗震性能的元件; (3)对于高层建筑的设计, 可以尽 量采用电缆桥架的方式连接线路, 这样可以降低下层的线路数量。总之, 只有综合设计符合环境的设备体系, 才可以最大程度的保障设备的使用质 量以及性能。

\section{2 元件排布优化方案}

在设备的运行中, 元件的排布是设备的重要组成部分, 需要经过不断 的优化设计来提升性能。

\section{2. 1元件结构形式的优化}

在设备元件结构形式的优化中, 需要保证安装高度与柜底之间的间距 在 $20 \mathrm{~cm}$ 以上, 以便可以保证设备的维修效率; 对于操作手柄的设计, 需要距 离柜底 $1.5 \mathrm{~m}$ 左右; 在仪表的设计中, 需要控制在视线可及的范围内; 对于 具有热量散发的元件, 需要添加散热装置; 例如在大电流及负荷的元件设 计优化中, 其线路相对较多, 在设计过程中, 需要将柜体设计在位置较低处, 以便可以提升布线的效率。另外, 在设计的过程中, 要考虑建筑的整体结构 形式, 从而对线路的排布以及柜体的安装进行合理的结构优化, 保障最佳 的性能。

\subsection{2符合柜体的排布设计}

在元件的布置优化中, 需要根据柜体的形式来进行优化设计, 如在抽 庶类的柜体设计中, 可以选择插拔式开关, 其可以提升设备的操作性。同时, 在元件的排布过程中, 要考虑整体的空间结构, 对于结构较为紧密的排布, 需要选择结构较小以及防护性能高的元件, 其可以提升系统的整体使用性 能。另外, 在元件的设计中, 需要保障拆卸位置的合理性。总之, 在设计的 过程中, 只有保证符合柜体的排布方式, 才可以最大程度的保障其性能。
2.3设备性能优化方案

2.3.1低压成套开关设备优化方案

在系统的优化方案中, 应该对低压成套开关设备进行优化, 需要根据 用户的使用量, 设置合理的载流容量, 以便最大程度的保证设备的使用性 能。另外, 在空气污染较为严重或者湿度相对较大的地区, 需要设置抵抗电 离以及密封措施, 降低元件的损坏。

2. 3. 2 高压成套开关设备优化方案

保护高压成套开关设备时, 需要保证柜体的结构强度, 使其在承担错 误操作时, 可以避免元件受到损伤; 在设计过程中, 需要保证程序锁以及闭 锁电磁铁可以进入条件限制。另外, 在设计过程中, 对于非标准柜体的设计, 需要在满足使用条件的基础上, 提升整体的承受能力, 这样可以避免安全 事故的发生率。

\section{4 采用新技术和新材料}

随着我国电力电子技术、控制技术、信息技术的发展, 我国电网逐渐 向自动化、智能化方向发展, 对高低压成套开关设备的性能要求更高。因 此, 高低压成套开关设备进行优化设计的时候, 除了符合成套开关设备的 基本要求以及国家相关标准之外, 还有积极使用新技术、新材料、新工艺, 提升产品的性能, 最大限度提升开关设备的性能。将传感技术、遥信技术、 红外测温技术、智能配电监控模块、网络技术应用在成套开关设备中, 通 过网络接口连接中央控制室和设备, 从而实现对供配电系统的电压、电流、 有功功率、无功功率等参数进行监测, 并对断路器的状态进行控制, 通过远 程监控系统, 实现对开关设备的遥测、遥信、遥控以及遥调。计算机实时 采集高低压成套开关设备的电压、电流、有功功率、功率因素等信息, 并 对这些数据信息进行分析、处理、记录, 并自动生成报表, 为电气设备的维 修提供参考。通过对开关设备的运行状态、保护动作进行实时监控, 如果 发现设备运行存在异常, 系统会自动发出警报, 并实时记录各柜内开关的 状态、过电压、过电流以及跳闸等动作。通过分析开关设备的实时状态, 对开关设备的开合进行控制。目前我国高低压开关设备逐渐向网络化、智 能化、数字化、结构紧凑等方向发展, 它降低了设备维修和管理时间, 可以 实现开关设备的定期检查、在线监测、远程控制、故障诊断等功能, 实现 开关设备的智能化控制。

\section{3 结语}

在电力工程中高低压成套开关设备占据着十分重要的地位, 它的性能 直接关系到能否实现稳定配送电以及居民用电安全、稳定。从目前来看, 我国高低压成套开关设备生产企业众多,已超过两万家。随着社会的不断 发展, 对高低压成套开关设备的要求也将越来越高。

\section{[参考文献]}

[1]曹凯媇.高低压成套开关设备的优化设计[J].建筑工程技术与设 计,2018,(3):2359.

[2]赖美云.高低压成套开关设备的优化设计解析 [J].中国设备工 程,2017,(16):188-190.

[3]王艳霞.高低压成套开关设备的优化设计解析 [ J]. 商品与质 量,2018,(15):287. 